\title{
Design of a Rotary Sprout Harvester
}

\author{
GUO Yi ${ }^{a}$, LIU Jiwei ${ }^{\text {b, * }}$
}

Department of Horticulture, Beijing Vocational College of Agriculture, Beijing, 102442, China

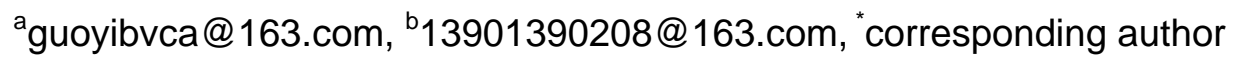

Keywords: Rotary Blade; Harvester; Sprout; SolidWorks; Matlab

\begin{abstract}
In order to solve the issues such as high labor intensity, low work efficiency as well as bad reaping quality regarding manual sprout harvesting, realization of feeding of sprouts by self-weight for the existing sprout harvester and manual collection required for reaped sprouts, a type of rotary sprout harvester has been developed. This article, based on SolidWorks software, sets up 3D models of parts and complete unit of the rotary sprout harvester, the mathematical model of kinematic analysis of the cutter of double eccentric circular cam mechanism, and draws variation curve of displacement, speed and acceleration by Matlab. As for the rotary sprout harvester based on this design, the results of the application tests show that time for single round of reaping is 12s, which compared with manual reaping, increases work efficiency by more than $300 \%$ with fine harvesting quality; via the transmission device and the feed box, automatic connected sprout feeding and collection of reaped sprouts are realized. If the rotary sprout cutter in this design is applied to sprout reaping, labor intensity can be reduced, work efficiency increased and operation quality guaranteed. Besides, as such mechanism is characterized by simple structure, convenient operation, safety and low manufacturing cost, it solves the issue of mechanization of sprout reaping, thus easy to be promoted in production.
\end{abstract}

\section{Introduction}

With the development of the society, people have started to pursue a healthy ecology instead of the previous basic needs connected with vegetables. People now pay more attention to index of vegetables like quality and appearance, having increasing demands for health-care and functions of vegetables. Sprouts are fresh and tender with unique flavor and free from pollution. It also contains varied amino acids, minerals and vitamins as well [1]. Sprouts have congestion-alleviating, anti-inflammatory, hypolipidemic and anti-hypertension effects as well, having gained increasing favors and attentions from the consumers and the markets and thus being placed in the first class of vegetables of fashion and specialty [2].

As a kind of convenient and practical three-dimensional modeling software, SolidWorks has a powerful CAE function, which is convenient for the designer to check the design parameters and optimize the design models in the design stage. Wang Fang, et al. applied SolidWorks's disc cam for the toothed drum-type pickup device of the forage harvester to conduct parametric design [3]. Liu Zhigang, et al. applied SolidWorks to conduct 3D modeling design of all parts of the pepper picker [4]. Xu Qingqing, et al. completed 3D modeling of the stalk crushing and forming machine [5]. Zheng Lei, et al. conducted the structural improvement of the crawler-type harvester chassis frame [6]. Guo Yi, et al. completed the design of the atomizing cultivation device for sprouts [7]. Su Jianwei, et al. designed the vibrating clamp for muskmelon grafting machine [8]. Tang Ningning, et al. designed and optimized the flail knife of the horizontal banana straw chopper [9].

As one of the three most widely applied mathematical software today, Matlab can also be used to draw images in addition to Matlab's remarkable function of numerical and matrix computing. Wang Jinwu, et al, based on Matlab, conducted the reverse design as well as kinematic analysis for the planetary pricking hole mechanism for the irregular gear [10]. Wu Kang, based on Matlab, conducted the analysis and simulation of motion of the blade of the caragana cutter [11]. Yang Chunmei, et al., based on Matlab, conducted the design of optimization of the drive system of the cutting mechanism for small shrubs [12]. Zhang Hongjun, et al., based on Matlab, conducted the design of optimization 
of cleaning mechanism of the corn sheller [13]. Zhao Huahui, et al., based on Matlab, conducted the analysis of simulation of motion of the rotary cultivator [14].

So far, sprout reaping mainly depends on hand scissors with low work efficiency, high labor intensity and great difficulty in guaranteeing reaping quality. Guo Yi has developed a kind of sprout harvester, in which sprout feeding is achieved by self-weight and the reaped sprouts should be collected by men. Thus, sprout reaping and collection require 2 persons who work simultaneously [15]. This paper is designed to introduce a kind of sprout harvester with rotary blades which is characterized by a simple structure, easy operation, safe use and low manufacturing cost. This harvester realizes automatic linked feeding of sprouts as well as collection of reaped sprouts via the transport unit and feed box. As this harvester only requires one person to complete sprout reaping, it reduces labor intensity of sprout harvesting, increases work efficiency and guarantees operation quality.

\section{General Construction and Working Process}

The rotary sprout harvester is made up of the frame, the transport unit, the reaping unit, feed box, the slide and the control system (Fig.1). For the purpose of ensuring that the harvested sprouts fall into the feed box in a smooth manner, the inclined structure is adopted for the frame. The delivery unit is installed on one side of the frame. One terminal of the conveyor belt is used for the feeding of the sprouts to be reaped and the other terminal for importing of the seedling tray for the harvested sprouts. The reaping unit is installed on the other side of the frame for the sprout reaping. The hopper is installed on the lower part of the reaping unit to collect the reaped sprouts. The slide is installed upon the end frame on the transport side of the conveyor belt to assist falling of the seedling tray.

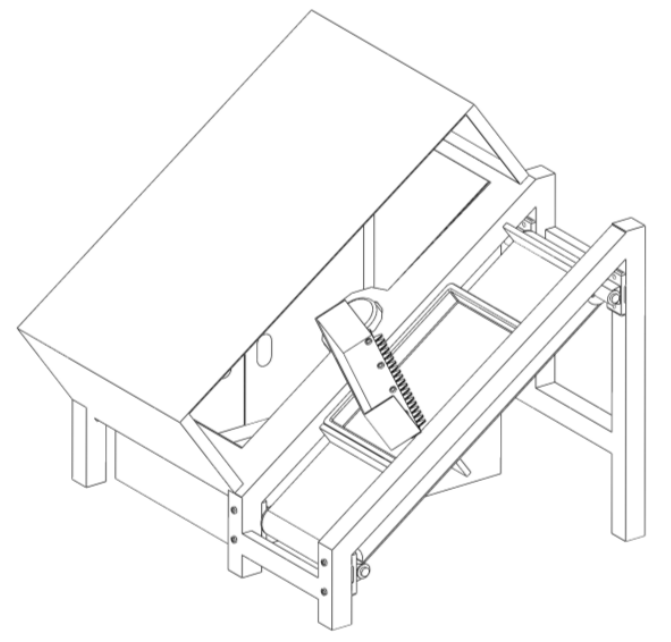

Fig. 1 Sketch of the reciprocating device

Working process of the reaper is as follows: First, start the control system of the reaper to enable the conveyor belt and the cut-off unit to work. Then, place the sprouts to be reaped together with the seedling tray in the direction of the feeding for the conveyor belt. As the sprouts to be reaped reach the location of the cutter so as to complete sprout harvesting, the harvested sprouts, pushed by the feeding plate of the cutter, reach the upper side of the feed box with rotation of the cutter and fall into the box. In the meantime, the conveyor belt delivers the seedling tray to the slide and the tray falls. The other sprouts to be reaped are delivered to the cutter to be reaped. By following such procedure repeatedly, continuous harvesting of sprouts is realized.

\section{Design of Key Parts}

Design of the Frame. For the convenience of the reaped sprouts to fall into the feed box smoothly, the inclined structure is adopted for the frame. The angle of inclination is $45^{\circ}$. Based on the principles of ergonomics, in order to reduce labor intensity, the operator works in a seated position. The height of the frame in the operation spot is designed as $700 \mathrm{~mm}$. The frame is made of stainless steel 
materials which are welded together. The design of the frame is completed with the welding tools provided by SolidWorks software. See Fig. 2.

Design of the Delivery Unit. The delivery unit includes the drive motor, the conveyor belt, the driving roll, the driven roller and the support roll (Fig. 3). It is installed on one side of the frame. In order to ensure that feeding rate does not change with weight during sprout reaping and delivery of the seedling tray, partitions are designed on the conveyor belt. In order to avoid the disturbance between feeding and reaping, the spacing between partitions is designed as $500 \mathrm{~mm}$.

Design of the Reaping Unit. The reaping unit is made of the transmission box, the cutter and the electric motor (Fig.4).The double-blade cutter is adopted. It consists of the upper blade and the lower blade. See parameters of the structure in Table 1. During work hours, the upper and lower blades, driven by the twin cam inside the transmission box, are in a relative motion so as to realize cutting of sprouts in the location of the blade teeth. Such cutting is done in shearing pattern so as to effectively guarantee a trim cut. The transmission box, getting power from the motor, on one hand, drives the cutter as a whole to move in a circle, on the other hand, drives the cutter to work in relative reciprocating motion to realize sprout harvesting and delivery of the reaped sprouts to the feed box. Structural parameters of the cut-off unit are shown in Table 1.

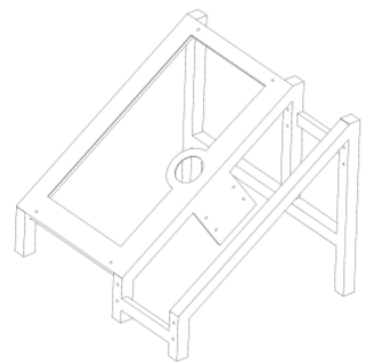

Fig. 2 Frame Fig.

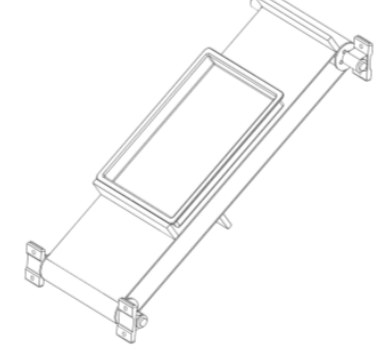

3 Delivery unit Fig.

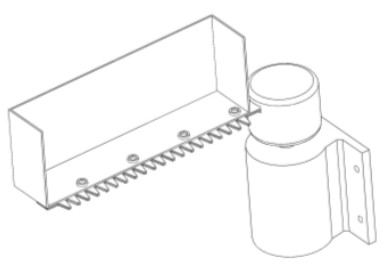

4 Reaping unit Structural Parameters $200 \mathrm{~mm} * 200 \mathrm{~mm} * 170 \mathrm{~mm}$ $345 \mathrm{~mm} * 20 \mathrm{~mm} * 2 \mathrm{~mm}$

\begin{tabular}{|c|c|c|}
\hline Transmission box & Length*width*height & 200mm*200mm*170mm \\
\hline \multirow{7}{*}{$\begin{array}{l}\text { Upper blade\& lower } \\
\text { blade }\end{array}$} & Length*width*thickness & $345 \mathrm{~mm} * 20 \mathrm{~mm} * 2 \mathrm{~mm}$ \\
\hline & Teeth height & $15 \mathrm{~mm}$ \\
\hline & Number of teeth & 20 \\
\hline & Space width & $13 \mathrm{~mm}$ \\
\hline & Addendum width & $3 \mathrm{~mm}$ \\
\hline & Addendum width & $10 \mathrm{~mm}$ \\
\hline & Cutting edge angle & $76.9^{\circ}$ \\
\hline Fixed film & Length*width*thickness & $288 \mathrm{~mm} * 20 \mathrm{~mm} * 2 \mathrm{~mm}$ \\
\hline Baffle & Height & $100 \mathrm{~mm}$ \\
\hline
\end{tabular}

Design of the Control System. The control system is mainly made up of button switch and leakage protector for the purpose of start and stop of the machine.

\section{Matlab-based Kinematic Analysis of the Cutter}

Set up Mathematical Model for Kinematic Analysis of the Cutter. The kinematic principle for the cutter is that the double-eccentric circular-cam mechanism realizes cutting of the upper and lower blades via reciprocating motion of the upper blade driven by the upper cam and of the lower blade driven by the lower cam. Kinematic analysis of the mechanism is conducted by the analytic method. First, establish displacement equation of the mechanism, and then get derivative of displacement equation with respect to time to get velocity equation of the mechanism. Then, get derivative of speed equation with respect to time to get acceleration equation of mechanism so as to complete kinematic analysis of mechanism.

According to the definite structure of the cutter, draw a sketch of kinematic analysis on the upper cam mechanism. See Fig.5. See Fig. 6 for the sketch of kinematic analysis on the lower cam 
mechanism. Set up the mathematical model of kinematic analysis respectively. Table 2 shows relevant parameters.

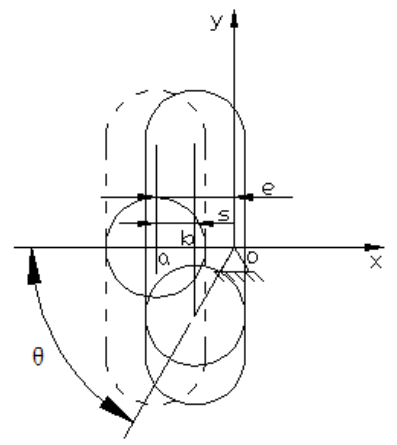

Fig.5 Upper cam mechanism

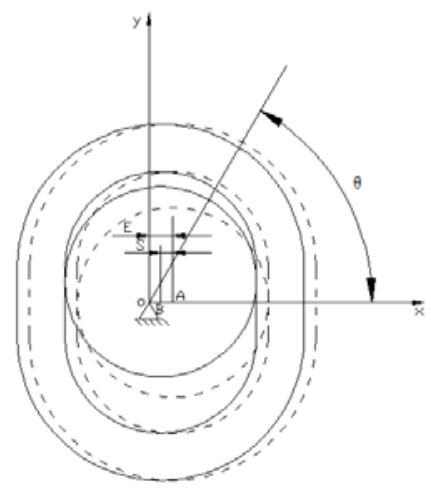

Fig.6 Lower cam mechanism

Analytical mathematical model for the upper blade based on Fig. 5 is as follows:

Displacement of upper blade:

$$
s=e-e \cdot \cos \omega t
$$

Speed of the upper blade:

$v=e \cdot \omega \cdot \sin \omega t$

Acceleration of upper blade:

$a=e \cdot \omega^{2} \cdot \cos \omega t$

Analytical mathematical model for the lower blade based on Fig. 6 is as follows:

Displacement of lower blade:

$S=-E+E \cdot \cos \omega t$

Speed of the lower blade:

$V=-E \cdot \omega \cdot \sin \omega t$

Acceleration of lower blade:

$A=-E \cdot \omega^{2} \cdot \cos \omega t$

Table 2 Description of Relevant Parameters

\begin{tabular}{|c|l|}
\hline Sign & \multicolumn{1}{|c|}{ Meaning } \\
\hline$s$ & Displacement of upper blade \\
\hline$v$ & Speed of the upper blade \\
\hline$a$ & Acceleration of upper blade \\
\hline$e$ & Upper cam eccentricity \\
\hline$S$ & Displacement of lower blade \\
\hline$V$ & speed of the lower blade \\
\hline$A$ & Acceleration of lower blade \\
\hline$E$ & Lower cam eccentricity \\
\hline$\theta$ & Cam angle $\theta=\omega t$ \\
\hline$\omega$ & Camshaft rotation angular velocity \\
\hline
\end{tabular}

As for this design, DC reduction motor whose speed $n$ is $5 r / m i n$ is chosen to supply power. Camshaft rotation angular velocity $\omega$ is $0.52 \mathrm{rad} / \mathrm{s}$. Upper cam eccentricity e is $2.5 \mathrm{~mm}$. Lower cam eccentricity $\mathrm{E}$ is $4 \mathrm{~mm}$.

Kinematic analysis of the cutter based on Matlab. Upon kinematic analysis based on Matlab software, first, set up the mathematical model of kinematic analysis of the blade based on the above description, make programs, enter the specific numbers of relevant parameters, obtain kinematic parameters of displacement, speed and acceleration via programs, and draw variation curve of 
displacement, speed and acceleration by Matlab’s drawing function (Fig.7). The results of kinematic analysis show that the upper blade and the lower blade at any cam angle move in opposite direction with speed and acceleration in opposite directions, thus cutting can be realized. The structural parameters of this cutter are designed in a reasonable way.
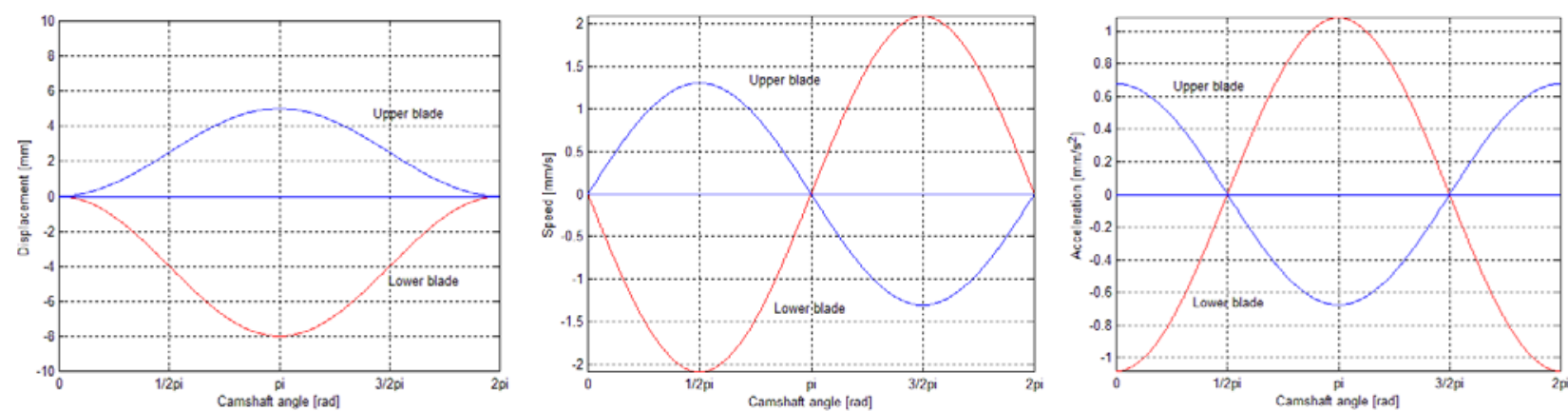

Fig. 7 Results of kinematic analysis of the cutter

\section{Conclusions}

This article, based on SolidWorks software, sets up 3D models of parts and complete unit of the rotary sprout harvester, the mathematical model of kinematic analysis of the cutter of double eccentric circular cam mechanism, and draws variation curve of displacement, speed and acceleration by Matlab.

As for the rotary sprout harvester based on this design, the results of the application tests show that time for single round of reaping is $12 \mathrm{~s}$, which compared with manual reaping, increases work efficiency by more than 300\% with fine harvesting quality; via the transmission device and the feed box, automatic connected sprout feeding and collection of reaped sprouts are realized.

If the rotary sprout cutter in this design is applied to sprout reaping, labor intensity can be reduced, work efficiency increased and operation quality guaranteed. Besides, as such mechanism is characterized by simple structure, convenient operation, safety and low manufacturing cost, it solves the issue of mechanization of sprout reaping, thus easy to be promoted in production.

\section{Acknowledgements}

This paper is co-funded by "Demonstration and Promotion Project of the Sprout Harvester" (Project No. XY-YF-16-01) and the "Cultivation Technology Research and Demonstration Project of Special Leaf Vegetables like Ice Plants" (Project No. XY-YF-17-01) of Technology Development and Demonstration and Promotion Fund of Beijing Vocational College of Agriculture.

\section{References}

[1] Guo Yi, Chen Lanfen, Zhang Guiqin. Design of a Sprout Layer-rack Aeroponic Cultivation Device[J]. Advances in Engineering Research, 2016, (104): 395-400.

[2] Guo Yi, ZHANG Guiqin. Development of A Design System for the Sprout Vertical Cultivation Device[J]. Advances in Engineering Research. 2015, (26):206-211.

[3] WANG Fang, WANG Hongming, XIAO Zixue, XING Jihui, AO Encha. Parametric Design and Kinematics Simulation on Spring-Finger Cylinder Pickup Collector[J]. Journal of Agricultural Mechanization Research, 2017, (05):57-62.

[4] LIU Zhigang, ZHAO Xiaoyan. Structure Design and Motion Simulation of High Branch Pepper Picking Device Based on SolidWorks[J]. Machine Building \& Automation, 2017, (02):130-132.

[5] XU Qing-qing, CHEN Ying. Design Study on the Ring Pattern Straw Crushing and Briquetting All-in-One Machine[J]. Mechanical Research \& Application, 2017, (01):83-84+87. 
[6] ZHENG Lei, ZHOU Hai, ZENG Yong, FENG Yong. Static Analysis and Structure Improvement of the Chassis Frame of Certain Crawler-Type Harvester[J]. Machinery Design \& Manufacture, 2017, (03):228-232.

[7] Guo Yi, Chen Lanfen, Suo Lang Jin Zong, Yang Zhen. Design of Sprout Aeroponics Equipment[J]. Advances in Engineering Research, 2016, (88): 1783-1789.

[8] SU Jianwei, LI Yangguang. Design of Attached Melon Grafting Machine Vibration Clamp Device[J]. Agricultural Equipment \& Vehicle Engineering, 2017, (03):30-34.

[9] TANG Ningning, LI Yue, LIANG Dong, ZHANG Xirui, ZHOU Teng, SONG Yating, WU Sihao, WANG Ziqiang. The Design and Optimizing on Flail Knife of Horizontal Banana Straw Crushing-returning Machine[J]. Journal of Agricultural Mechanization Research, 2017, (04):107-111+116.

[10]WANG Jinwu, ZHANG Chunfeng, ZHOU Wenqi, PAN Zhenwei, TANG Han, DUO Tianyu. Optimization design of non-circular planetary gear trains pricking hole mechanism based on Matlab [J]. Transactions of the Chinese Society of Agricultural Engineering (Transactions of the CSAE), 2016, 32(3): 22-28.

[11]WU Kang. Korshinskii cutting machine tool motion analysis based on Matlab simulation[D]. Shanxi Agricultural University, 2015.

[12] YANG Chunmei, GUO Minghui, Matlab Yan, LIU Qianjun, YANG Tie. Optimization Design of the Transmission System of Small-size Shrub Brush Cutter based on Matlab[J]. Journal of Mechanical Transmission, 2017, (03):78-82.

[13]ZHANG Hongjun, CHANG Chuandong, LIU Meng. Optimization Design of Cleaning Mechanism in Corn Thresher Based on MatLab[J]. Journal of Agricultural Mechanization Research, 2017, (08):102-106+111.

[14]ZHAO Hua-hui, LI Yun-wu, ZENG Qing-qing, LIU De-xiong. Imulation of rotary tiller based on Matlab[J]. Journal of Northwest A \& F University(Natural Science Edition) , 2016, (01):230-234.

[15]Yi Guo, Song Dong, Subo Tian. Design on Control System of Seedling Vegetable Harvester Based on Single Chip[J]. Advanced Materials Research, 2013, (721): 656-660. 